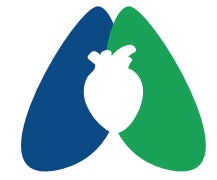

ASSOBRAFIR

C I Ê N C I A

\title{
A nova Diretriz Brasileira de Reabilitação Cardiovascular reflete de fato a multidisciplinaridade?
}

\author{
Does the new Brazilian Cardiovascular Rehabilitation Guideline \\ really reflect multidisciplinarity?
}

Rafael Michel de Macedo' (D); Audrey Borghi-Silva² (D); Pedro Dal Lago ${ }^{3}$ (D); Aparecida Maria Catai ${ }^{2}$ (1); Giulliano Gardenghi ${ }^{4,5,6,7}$ (1); Michel Silva Reis ${ }^{8}$ (1)

Após a leitura atenta e, com grande interesse, no recente artigo publicado nos Arquivos Brasileiros de Cardiologia', que tem como proposta atualizar a Diretriz Brasileira de Reabilitação Cardiovascular, nos causou enorme surpresa a forma como foram descritos muitos aspectos relacionados ao tema tão relevante e de abordagem multidisciplinar. De acordo com os próprios autores, trata-se de um documento que tem como objetivos "informar e não substituir o julgamento clínico do médico, que, em última análise, deve determinar o tratamento apropriado para seus pacientes". Contudo, uma Diretriz que trata de um assunto amplo, complexo, multidisciplinar, envolvendo uma equipe de cuidados, como é o caso da Reabilitação Cardiovascular (RCV), deveria prezar pelos seus pressupostos fundamentais. Desta forma, consideramos que deve ser papel daquele documento, orientar e nortear as ações inerentes não somente do profissional médico, mas também a de todos os demais profissionais envolvidos na RCV.

Neste contexto, entendemos que falta ao documento abrangência e

${ }^{1}$ Academia do Coração, Hospital Cardiológico Costantini, Curitiba, PR, Brasil

2Programa de Pós-graduação em

Fisioterapia (PPGFt), Departamento de Fisioterapia, Universidade Federal de São Carlos (UFSCar), São Carlos, SP, Brasil 3Programa de Pós-graduação em Ciências da Reabilitação, Universidade Federal de Ciências da Saúde de Porto Alegre (UFCSPA), Porto Alegre, RS, Brasil

${ }^{4}$ Hospital Encore, Aparecida de Goiânia, GO, Brasil

${ }^{5}$ Faculdade Ceafi, Goiânia, GO, Brasil ${ }^{6}$ Lifecare, Unidade de Terapia Intensiva em Queimados, Hospital Estadual de Urgências Governador Otávio Lage de Siqueira (HUGOL), Goiânia, GO, Brasil

${ }^{7}$ Hospital e Maternidade São Cristóvão, São Paulo, SP, Brasil

${ }^{8}$ Grupo de Pesquisa em Avaliação e Reabilitação Cardiorrespiratória (GECARE), Faculdade de Fisioterapia, Universidade Federal do Rio de Janeiro (UFRJ), RJ, Brasil

Como citar: Macedo RM, Borghi-Silva A, Dal Lago P, Catai AM, Gardenghi G, Reis MS. A nova Diretriz Brasileira de Reabilitação Cardiovascular reflete de fato a multidisciplinaridade? ASSOBRAFIR Ciênc. 2020;11:e42205. https://doi. org/10.47066/2177-9333.AC.2020.0011

Submissão em: Novembro 30, 2020 Aceito em: Fevereiro 04, 2021

Estudo realizado em: Universidade Federa de São Carlos (UFSCar), São Carlos, SP Brasil.

Aprovação ética: não há.

*Autor correspondente:

Audrey Borghi-Silva. E-mail: audrey@ufscar.br detalhamento de ações reconhecidamente multidisciplinares, e não somente as médicas. Como consequência, em vários momentos no texto, perde-se o foco e a abrangência, pois uma Diretriz tem como seus pilares fundamentais direcionar as ações de todos os envolvidos no processo. Além disso, fica claro na leitura do documento, que há uma completa dissociação quanto a correta conceituação do que se entende mundialmente como RCV, e o Brasil não pode ser excluído do contexto Internacional nesta área.

Revisitando estes conceitos, a Reabilitação é definida pela Organização Mundial de Saúde (OMS) como um conjunto de medidas que auxiliam aqueles que perderam função a readquiri-la ${ }^{2}$. Especificamente, a RCV é entendida como um importante componente no cuidado continuado de indivíduos com doenças cardiovasculares, fornecendo um programa multidisciplinar de educação e exercícios físicos, suporte psicológico e social com o objetivo de diminuir a morbimortalidade e melhorar a qualidade de vida dos pacientes ${ }^{3,4}$.

Outrossim, o documento destaca de forma equivocada, os limites de prescrição da atividade física, a qual mostra-se subordinada a prescrição do profissional médico, que de forma não cristalina, parece desconhecer a legitimidade de outros profissionais da saúde, na sua capacidade para prescrever os exercícios físicos, o que é fundamental para a resposta positiva dos participantes dos programas de RCV.

Ademais, não podemos nos furtar do momento. Vivemos uma crise pandêmica, onde quem está na linha de frente tem percebido a importância da atuação multidisciplinar. Além disso, a crise financeira noticiada diariamente e massivamente veiculada por periódicos científicos e pela imprensa constrói um cenário desfavorável para programas de RCV no país (maioria custeada pelos pacientes) $)^{5}$. Sendo assim, entendemos que é momento de união de todos os envolvidos no time de cuidados no país e, sobretudo, pela necessidade de proporcionar cuidado integral para os pacientes. Entendemos que existem dois 
caminhos a seguir, o primeiro do qual concordamos é criar uma legítima diretriz nacional, com foco na multidisciplinaridade, na qual demais profissionais possam incorporar seu conhecimento, proporcionando aos leitores um documento completo, que pode servir a todos os profissionais que atuam na linha de frente, ou o segundo partindo para total fragmentação onde cada especialidade criará a sua diretriz fragmentado as ações do cuidado. Enfim, a discussão está posta, quem sabe a atual crise pode ser um marco para uma nova forma de pensamento de RCV no país.

Portanto, deve ser objeto de consideração, que existe, e, sempre existirá desconforto por parte de diferentes profissionais da área da saúde, que fazem parte da equipe multiprofissional e trabalham com reabilitação, enquanto houver documentos publicados sobre reabilitação nos quais diferentes componentes da equipe, não se encontram inseridos no processo de construção e atuação na assistência aos pacientes com doença cardiovascular. Reforçamos aqui, que este descompasso é a principal motivação desta carta ao editor.

\section{FONTE DE FINANCIAMENTO}

Conselho Nacional de Desenvolvimento Científico e Tecnológico - CNPq. AMC, ABS e PDL são investigadores estabelecidos do CNPq I (nível 1A, IB e ID, respectivamente).

\section{CONFLITO DE INTERESSES}

Nada a declarar.

\section{REFERÊNCIAS}

1. Carvalho T, Milani M, Ferraz AS, Silveira AD, Herdy AH, Hossri CAC, et al. Diretriz Brasileira de Reabilitação Cardiovascular-2020. Arq Bras Cardiol. 2020;114(5):943-87. http://dx.doi.org/10.36660/abc.20200407. PMid:32491079.

2. WHO: World Health Organization. Relatório mundial sobre deficiência [Internet].Switzerland: WHO; 2011. (cap. 4. Rebilitação) [citado em 2020 Nov 30]. Disponível em: https:// www.who.int/disabilities/world_report/2011/chapter4_por. pdf?ua=1

3. Price KJ, Gordon BA, Bird SR, Benson AC. A review of guidelines for cardiac rehabilitation exercise programmes: is there an international consensus? Eur J Prev Cardiol. 2016 Nov;23(16):1715-33. http://dx.doi. org/10.1177/2047487316657669. PMid:27353128.

4. Thomas RJ, Beatty AL, Beckie TM, Brewer LC, Brown TM, Forman DE, et al. Home-Based Cardiac Rehabilitation: a Scientific Statement From the American Association of Cardiovascular and Pulmonary Rehabilitation, the American Heart Association, and the American College of Cardiology. Circulation. 2019 Jul 2;140(1):e69-89. http://dx.doi. org/10.1161/CIR.0000000000000663. PMid:31082266.

5. Pesah E, Turk-Adawi K, Supervia M, Lopez-Jimenez F, Britto $\mathrm{R}$, Ding R, et al. Cardiac rehabilitation delivery in low/middleincome countries. Heart. 2019 Dez;105(23):1806-12. http:// dx.doi.org/10.1136/heartjnl-2018-314486. PMid:31253695. 